Motive fehlen, bleibt das Spendenaufkommen (nach Wegfall jeglicher Steuerbegünstigung) sehr gering. Das empirische Ergebnis wissenschaftlicher Analyse erklärt den „überraschenden" Befund deutscher Journalisten. ${ }^{2}$

Zur Reform von Parteienfinanzierungsregimen (in real existierenden Rechtsstaaten) wird vor allem die Offenlegung von Spenden empfohlen, weil sich so das politische Risiko für Geldgeber und -nehmer beträchtlich erhöht. Verbote oder Höchstgrenzen für Unternehmensspenden hält der Autor für empirisch unbegründet. Die Väter und Mütter des Grundgesetzes haben also mit ihrem Publizitätsgebot (ohne Verbote und Beschränkungen) offenbar instinktiv alles richtig gemacht.

Nach dem heutigen Stand hat Iain McMenamin zum Thema Parteienfinanzierung das wichtigste Buch des Jahrzehnts geschrieben. Bemerkenswert ist vor allem die Kaltblütigkeit, mit der er auf strikt empirischer Basis ein altes Gespenst - den weithin akzeptierten Satz: „Politik ist immer und überall durch Spenden bestechlich“ - zu Grabe trägt.

Die einzige Schwäche seines Buches liegt in der lückenhaften bibliographischen Vorarbeit. So sind ihm zahlreiche Beiträge einer Vielzahl von Autoren aus dem Umfeld des IPSA Research Committee „Political Finance and Political Corruption“ unbekannt geblieben: Je zwei Bände von Herbert E. Alexander (1979, 1989) und Arnold J. Heidenheimer (1968, 1970), Arbeiten von Colin Hughes (Australien), Uwe Schleth, Arthur B. Gunlicks und Peter Lösche (alle zu Deutschland), aber auch die klassischen Studien von Michael Pinto-Duschinsky über die britische Politikfinanzierung und die deutschen Parteistiftungen ebenso wie die zahlreichen Aufsätze von Khayyam Z. Paltiel (1972 bis 1986).

Karl-Heinz Naßmacher

2 „Weniger Großspenden an Parteien“, in: Nordwest-Zeitung vom 31. Dezember 2014, http://www. nwzonline.de/politik/weniger-grossspenden-an-parteien_a_22,0,831683074.html; Philip Plickert, Großspenden sind nur Kleingeld für die Parteien, in: FAZ vom 24. Dezember 2014, http://www. faz.net/aktuell/wirtschaft/verdeckte-parteienfinanzierung-grossspenden-sind-nur-kleingeld-fuerdie-parteien-13339408.html; Andreas Maisch, Den Parteien brechen die Großspender weg, in: Die Welt vom 22. November 2014, http://www.welt.de/politik/deutschland/article134617609/DenParteien-brechen-die-Grossspender-weg.html (jeweils Abruf am 14. Januar 2015).

\title{
Die Beziehungen von Parteien und Gewerkschaften: Informationsfülle mit Theoriedefiziten
}

Neusser, Christian: Pluralisierte Partnerschaften. Über den Wandel der Parteien-GewerkschaftsBeziehungen (Reihe: Forschung aus der Hans-Böckler-Stiftung, Mitbestimmung und wirtschaftlicher Wandel, Bd. 159), edition sigma, Berlin 2013, 350 Seiten, € 24,90.

Im Gefüge der Beziehungen zwischen Parteien und Verbänden in Deutschland hat die historisch gewachsene enge Partnerschaft zwischen SPD und Gewerkschaften über Jahrzehnte eine bedeutende, die soziopolitischen Konfliktlinien in Deutschland maßgebend prägende Rolle gespielt. Mit der zunehmenden programmatischen und wahlstrategischen Orientierung der SPD hin zur bürgerlichen Mitte setzte jedoch ein schleichender Entfremdungsprozess zwischen den beiden Organisationen ein, der durch die Agenda-Politik von Bundeskanz- 
ler Gerhard Schröder in den Jahren ab 2003 einen vorläufigen Höhepunkt erlebte. Diesen Trend, der sich zwischenzeitlich abgemildert, aber bis heute nicht umgekehrt hat, nimmt Christian Neusser zum Anlass für eine umfassende und systematische Analyse der Beziehungen zwischen Gewerkschaften und Parteien in Deutschland zwischen 1998 und 2009.

Es geht ihm darum, die verschiedenen Facetten der Beziehungen des DGB und seiner Einzelgewerkschaften zu den vier von ihm so genannten Sozialstaatsparteien CDU, SPD, Die Linke und Bündnis 90/Die Grünen sowohl in systematischer als auch in historischer Vergleichsperspektive umfassend zu beschreiben und deren Wandel mit den Veränderungen der politischen Rahmenbedingungen, insbesondere des Parteiensystems, der Gewerkschaften und des Sozialstaats, in Verbindung zu bringen. Ausgehend von der Arbeitshypothese, dass aus der ehemals „privilegierten Partnerschaft“ zwischen SPD und Gewerkschaften mittlerweile „pluralisierte Partnerschaften“ zwischen den Gewerkschaften und den vier Sozialstaatsparteien geworden seien, untersucht Neusser diese Beziehungsmuster in so verschiedenen Dimensionen wie der Sozialstruktur der Mitglied- und Wählerschaft, der Programmatik und Politik sowie der personellen und organisatorischen Verknüpfungen.

Neussers Analysen zufolge hat die mit dem Godesberger Programm einsetzende politisch-programmatische Modernisierung der SPD hin zur Volkspartei zu einer Verminderung der sozialstrukturellen Gemeinsamkeiten mit den Gewerkschaften und dies wiederum zu einer weiteren inhaltlichen Entfremdung zwischen beiden Organisationen geführt. Neusser zeigt zum einen exemplarisch am Beispiel der Sozialpolitik auf, welchen Schwankungen und Belastungen das Verhältnis zwischen SPD und Gewerkschaften im Zeitverlauf ausgesetzt war; er macht zum anderen aber auch deutlich, wie stark die beiden Organisationen trotz des fortschreitenden sozialstrukturellen Entkoppelungsprozesses auf Wählerund Mitgliederebene nach wie vor durch Doppelmitgliedschaften sowie durch institutionalisierte Kooperationsformen miteinander verbunden sind. Im Vergleich zur SPD zeichnen sich nach Neussers Analysen die Beziehungen zwischen den Gewerkschaften und der CDU durch eine größere Distanz, aber auch durch eine höhere Kontinuität aus, was nicht zuletzt in den relativ konstanten Wähleranteilen der Union unter den Gewerkschaftsmitgliedern zum Ausdruck komme. Trotz grundlegender programmatischer Differenzen in einigen zentralen wirtschafts- und gesellschaftspolitischen Fragen seien die strukturellen und personellen Verflechtungen auf Mitglieder- und Elitenebene bemerkenswert stabil geblieben.

Mehr Bewegung stellt Neusser demgegenüber bei den beiden jüngeren Sozialstaatsparteien, den Grünen und der PDS/Die Linke, fest. Die personellen und organisatorischen Verknüpfungen zwischen den Gewerkschaften und den Grünen seien eher lose und hätten sich, etwa was die Doppelmitgliedschaften von grünen Bundestagsabgeordneten angehe, seit der Gründungsphase der Partei sogar weiter abgeschwächt. Politisch-programmatisch sei jedoch seit den achtziger Jahren - durch die Abkehr der Grünen von einem radikalen Antiindustrialismus und durch die Öffnung der Gewerkschaften für ökologische Ziele eine Annäherung zwischen den beiden Organisationen zu verzeichnen. Gegen die PDS haben laut Neusser auf Seiten der Gewerkschaften trotz deren dezidiert gewerkschaftlich orientierter Programmatik lange Zeit Vorbehalte bestanden, die auf die Furcht vor einer Politisierung der Gewerkschaften, aber auch auf den von großen Teilen der Partei vertretenen entschiedenen Antikapitalismus zurückzuführen seien. Neusser zufolge ist der Partei dann aber durch die Verschmelzung mit der WASG zur Partei Die Linke ein beachtlicher Vorstoß in das gewerkschaftliche Milieu gelungen. Gleichwohl seien die programmatischen Schnittmengen mit den Gewerkschaften bis heute begrenzt geblieben. 
Insgesamt betrachtet besteht das Verdienst des Verfassers darin, eine Fülle von im Einzelnen vielfach sicher bekannten Informationen über die gewerkschaftliche Prägung der Wählerschaften, über die Sozialstruktur der Mitgliedschaften von Gewerkschaften und Parteien, über Doppelmitgliedschaften von Abgeordneten sowie über Sonderorganisationen der Parteien mit gewerkschaftlichem Bezug systematisch aufbereitet und vergleichend dargestellt zu haben. Darüber hinaus vermittelt Neusser - gestützt auch auf die Auswertung von Presseveröffentlichungen, Originaldokumenten und durch Experteninterviews - einen vertieften Einblick insbesondere in die politisch-programmtische Beziehungsdimension und die Elitenkontakte. Die teils sehr detaillierten Ausführungen über politisch-programmatische Übereinstimmungen und Divergenzen sowie über die personellen und organisatorischen Verknüpfungen zwischen den Gewerkschaften und den vier Sozialstaatsparteien sind mit großem Gewinn zu lesen. Demgegenüber ist es ein wenig bedauerlich, dass die zahlreichen interessanten und zum Teil originären Informationen nur in geringem Maße theoretisch gewürdigt werden. Neusser stellt die Parteien-Gewerkschafts-Beziehungen zwar in den theoretischen Kontext des Linkage-, des Machtressourcenansatzes sowie der Institutionentheorie und leitet daraus sein analytisches Konzept ab. Er verzichtet aber darauf, über die theoretische Fundierung des Forschungsprogramms hinauszugehen und nach strukturellen Erklärungen für die Art und die Richtung des Wandels in den Parteien-Gewerkschafts-Beziehungen zu fragen. Dabei hätte es nahegelegen, etwa der Frage nachzugehen, ob die partielle Entkoppelung zwischen SPD und Gewerkschaften auf Faktoren wie die Globalisierung, gewandelte Interessen oder den Elitenwandel beziehungsweise auf eine Kombination dieser Faktoren zurückzuführen ist. Außerdem weicht Neusser der sich geradezu aufdrängenden Frage aus, ob die SPD angesichts des Rückgangs des Anteils der gewerkschaftlich gebundenen Arbeitnehmer an der Wahlbevölkerung überhaupt eine strategische Alternative zu ihrer programmatischen Öffnung hin zu den Mittelschichten gehabt hätte, wenn sie das Ziel der Mehrheitsgewinnung nicht von vornherein aufgeben wollte. Nur in einem Fall stellt Neusser seine empirischen Befunde explizit in Beziehung zu bereits vorliegenden Forschungsergebnissen. Gegen die These von Thomas Poguntke über die anhaltende Bedeutung der Kollateralorganisationen von SPD und CDU erhebt er den empirisch gut belegten Einwand, dass die Arbeitsgemeinschaft für Arbeitnehmerfragen (AfA) bei der SPD und die Christlich-demokratische Arbeitnehmerschaft (CDA) bei der Union mit ihren ausgezehrten Basisstrukturen kaum noch die Funktion eines Scharniers zwischen Gewerkschaften und den beiden Parteien erfüllen könnten.

Die abschließende These, dass die Beziehungsmuster, die sich in den Nachkriegsjahrzehnten eingespielt hatten und in deren Zentrum die „privilegierte Partnerschaft“ der Gewerkschaften mit der SPD gestanden hatte, mittlerweile erheblich aufgeweicht und durch ein komplexeres Beziehungsgeflecht zwischen den Gewerkschaften und den vier Sozialstaatsparteien ersetzt worden seien, kann man gut nachvollziehen. Die schrumpfende gemeinsame sozialstrukturelle Basis bei Mitgliedern und Wählern und der Elitenwechsel bei den Parteien sind zwei in einer Reihe von Faktoren, die sicherlich zu einer Distanzierung der Gewerkschaften von den beiden Volksparteien beigetragen haben. Man kann Neusser nur zustimmen in seiner Einschätzung, dass die nachwachsende Generation von Parteienrepräsentanten eine weitgehend emotionslose Haltung zu den Gewerkschaften an den Tag legt, während diese umgekehrt zunehmend lobbyistisch agieren und mit den Parteien je nach politisch-programmatischer Opportunität wechselnde Bündnisse und Interessenkoalitionen eingehen. 